\title{
Paraplegia
}

\section{Semen Retrieval in Spinal Cord Injured Men}

\author{
H. B. Rawicki, MB, BS, FACRM, ${ }^{1}$ S. Hill, MB, BS, FACRM ${ }^{2}$ \\ ${ }^{1}$ Director Spinal Rehabilitation, Caulfield General Medical Centre, 260-294 Kooyong \\ Road, Caulfield 3162, ${ }^{2}$ Hampton Rehabilitation Hospital, Beach Road, Hampton, \\ Victoria, Australia.
}

\section{Summary \\ A fertility programme for spinal cord injured men has been in progress for nearly 5 years. Thirty eight men have been treated in this programme. Electroejaculation, vibration ejaculation and subcutaneous physostigmine have all been used successfully to obtain semen. Semen has been obtained from 21 of 24 men with a lesion at T8 or above, and from 4 of 11 men with lesions below T10. There have been 8 pregnancies from 6 couples. Key words: Electroejaculation; Vibration ejaculation; Subcutaneous physostig- mine; Fertility; Spinal cord injury.}

Infertility is a usual consequence of spinal cord injury (SCI) in men. With a complete lesion and without intervention only $1 \%$ of men are fertile. ${ }^{12}$ Collated reports suggest that without intervention approximately $14 \%$ of men with a complete lesion are able to ejaculate. ${ }^{13}$ Irrespective of whether ejaculation is aided or unaided semen quality is usually poor, and in particular sperm motility is low. ${ }^{4-6}$ Therefore, to significantly improve fertility in this group of men efforts must be made to improve the rate of semen collection as well as improving semen quality.

We have been running a fertility enhancement programme for SCI men since 1985. During this time we have seen 39 men, almost always with their partners, for the purpose of either directly intervening to improve fertility, or rarely to give these men some idea whether it is possible that they are fertile. This paper will concentrate on the results of our work with semen retrieval.

\section{Method}

All men were asked to try masturbation if they had not already done so, and in addition, where masturbation was unsuccessful a post masturbation sample of urine was examined looking for retrograde ejaculation before embarking on more invasive procedures. When masturbation failed intervention was undertaken.

There are now three safe and relatively easy methods for increasing semen retrieval. These are electroejaculation (EE), vibration ejaculation (VE) and subcuta- 
neous physostigmine (SP). EE was the first method of artificial ejaculation that we tried and remains one of the most successful methods. EE was first used by Horne in 1948 who obtained semen from 11 of 18 men. ${ }^{7}$ Our current equipment delivers a continuous sine wave current of 30 volts at $10 \mathrm{~Hz}$ through a rectal probe. This directly stimulates the sympathetic nerves to the seminal vesicles and ampulla of the vas deferens causing semen emission. ${ }^{8}$ For EE to be successful the T10-L1 spinal segments must be intact. Side effects included a high incidence of autonomic hyperreflexia $(\mathrm{AH})$ in those men with high lesions, and pain in those men with incomplete or low lesions. One of the authors (HBR) had performed EE on 3 men under general anaesthetic because of the pain problem.

VE entails a vibratory stimulus applied to the glans, frenulum or occasionally the base of the penis which the causes a reflex ejaculation rather than semen emission. ${ }^{9} 10$ Our equipment has been modified to vibrate at $80 \mathrm{~Hz}$. Ejaculation in our series has been preceded by erection in each case, whereas with EE this is not always so. Again, at least T10-L1 must be intact, and we have not tried VE in patients with lesions below T12. Side effects of VE included AH and in two men superficial trauma to the glans resulting in bruising in one man on one occasion, and a superficial ulcer in the other man also on one occasion.

$\mathrm{SP}$ is a development from intrathecal prostigmine which was initially used by Guttmann in the $1960 \mathrm{~s}^{4}$ but has been all but abandoned due to the unacceptable incidence of serious side effects. SP was first used by Chapelle ${ }^{11}$ in 1983. Physostigmine is a lipid soluble acetylcholinesterase inhibitor and thereby acts as a parasympathomimetic agent which is able to cross the blood-brain barrier. The parasympathomimetic activity within the spinal cord allows an increased sensitivity to reflex activity and hence improves success rates with either masturbation or VE. It is necessary to first give a peripherally acting anticholinergic agent (Buscopan 40 mg i.m.) to prevent severe cholinergic side effects. Again for this method to work the T10-Ll spinal segments must be intact. ${ }^{11}$

Side effects with SP include a high incidence of blurring of vision with the Buscopan (4/5 men), nausea and sometimes vomiting (2/5), and in 2 of the 5 men who have had this treatment 'marijuana like highs' that have lasted up to 1 hour.

We have now treated 35 men with different combinations of the above methods (4 men presented for information only). VE is always tried first as this is the simplest for couples to use unaided at home. Where this fails SP is tried and finally EE. We have only been using SP since April 1989 and although we did make some attempts with VE prior to 1989 it is only since January 1989 that there has been a consistent effort with this method.

Retrograde ejaculation has occurred in at least 4 patients. In two of these this problem has been overcome by tamponading the bladder neck with a $30 \mathrm{ml}$ catheter balloon during ejaculation. This has provided external ejaculation in both men. One other male, a very incomplete L5 paraplegic with an artificial urinary sphincter, found the urinary catheter very uncomfortable and preferred post ejaculation voiding (first alkalinising his urine) and semen retrieval following masturbation. His wife is now pregnant.

\section{Results}

The results are shown in the Table. Three men were able to achieve an external 
Table

\begin{tabular}{lccccc}
\hline Level & VE & SP & EE & MAS & Total \\
\hline C4-7 & $2 / 3$ & $2 / 2$ & $7 / 9$ & $1 / 13$ & $12 / 13$ \\
& & $(1 \mathrm{RE})$ & $(2 \mathrm{RE})$ & & \\
T3-8 & $1 / 4$ & $1 / 1$ & $6 / 7$ & $2 / 11$ & $9 / 11$ \\
C4-T8 & $3 / 7$ & $3 / 3$ & $13 / 16$ & $3 / 24$ & $21 / 24$ \\
T10-12 & $1 / 2$ & $1 / 2$ & $1 / 7$ & $1 / 10$ & $3 / 10$ \\
L5 & & & & $1 / 1$ & $1 / 1$ \\
Total & $4 / 9$ & $4 / 5$ & $14 / 23$ & $5 / 35$ & $25 / 35$ \\
& & & & & \\
\end{tabular}

Note that a number of patients have tried more than one method to achieve an ejaculate.

$\mathrm{RE}=$ Retrograde ejaculation

MAS $=$ Masturbation

ejaculate with masturbation prior to our initial consultation. From the Table it can be seen that with intervention there is a high chance of obtaining semen in men with lesions at T8 or above. Semen was obtained as an external ejaculate in 18 men and in a retrograde fashion in 3 men (total 21) of the 24 men in this group. Semen could be obtained from only 3 of 10 men with lesions T10-12. Each of these 3 men had an incomplete lesion. Fifteen couples have participated in a programme for 6 months or longer and as a result there have been 8 pregnancies from 6 couples including 1 using in vitro fertilisation and a donor (wife's sister) egg.

All men with lesions $\mathrm{C} 7$ or above suffered some degree of $\mathrm{AH}$. Where necessary (increase in $\mathrm{BP}>40 / 30$; systolic $\mathrm{BP}>150$; symptoms) this was treated prophylactically with either Labetolol $100 \mathrm{mg}$ p.o. or Nifedipine $10 \mathrm{mg}$ p.o. 30 minutes prior to ejaculation. This has been adequate treatment in all patients.

\section{Conclusion}

All three available methods of artificial ejaculation offer significant prospects of achieving an ejaculate. The level of the lesion is very important in determining the prospects of success in semen retrieval, and for patients with lesions above T10 and incomplete lesions below T10 semen retrieval is a realistic goal for use with artificial insemination. In these men the major objective must be to improve the quality of obtained sperm so as to further significantly improve prospects for fertility.

\section{References}

I David A, Ohry A, Rozin R. Spinal cord injuries. Male infertility aspects. Paraplegia 1977: 15: 11-14.

2 Amelar RD. Sexual function and fertility in paraplegic males. Urology 1982: XX(1):62-5.

3 Madduri et al. Plasma Androgens and estrogens in paraplegic men. Urology 1979: 13:179-181.

4 Guttmann L, Walsh JJ. Prostigmine assessment test of fertility in spinal man. Paraplegia 1971: 9: 39-50.

5 Brindley GS. The fertility of men with spinal injuries. Paraplegia 1984: 22:337-348.

6 Rawicki HB, Lording DW. Assisted fertility in complete paraplegia: Case report. Paraplegia 1988: 26:401-4.

7 Horne HW. Fertility Studies in Human Male with Traumatic Injuries. N Eng $\mathcal{f}$ Med 1948: 239: 959-961. 
8 Brindley GS. Electro-ejaculation. Its technique, neurological implications. Neurol Neurosurg Psych 1981: 44:9-18.

9 Szasz G. Vibratory stimulation of the penis in men with spinal cord. Paraplegia 1987: 25:60.

10 Brindley GS. Reflex ejaculation under vibratory stimulation in paraplegic men. Paraplegia 1981: 19: 299-302.

11 Chapelle PA et al. Neurological correlations of ejaculation and testicular size. $\mathcal{F}$ Neurol Neurosurg Psych 1988: 51:197-202.

12 Brindley GS. The fertility of men with spinal injuries. Paraplegia 1984: 22:337-348. 